\title{
Food combinatorics in the production of dehydrated products for a healthy diet
}

\author{
Victoria Strizhevskaya ${ }^{1 *}$, Marina Pavlenkova ${ }^{1}$, Nataliya $_{\text {Nosachyova }}{ }^{1}$, and Inna Simakova ${ }^{1}$ \\ ${ }^{1}$ Federal State Budgetary Educational Institution of Higher Education Saratov State Agrarian University named after N.I. Vavilov, \\ Saratov, Russia
}

\begin{abstract}
The aim of the study was to develop compositions of dehydrated products for a healthy diet using the principles of food combinatorics. Two types of snack products from plant materials were developed as objects of study. The study was performed by the calculation method - the nutritional value was calculated according to the tables of chemical composition and based on these recommendations on the use of vitamins for men and women (2 groups of labor intensity). Vitamins and biologically active substances (flavonoids) were analyzed by reverse phase HPLC. Dehydration was carried out using resonance IR drying. The selection of adaptogenic plant raw materials, the calculation of acceptable ratios made it possible to predict the replenishment of the needs of the human body for the following substances: vitamin A from 12 to $15 \%$, while the estimated amount of provitamins $A$, such as $\beta$-carotene and lycopene, compensates for this need significantly from 79 to $127 \%$ of daily allowance, vitamin K from $88 \%$ to $131 \%$, native dietary fiber from $35 \%$ to $51 \%$ of the recommended daily allowance, depending on the ratio of components in the predicted compositions.
\end{abstract}

\section{Introduction}

One of the main directions in the field of healthy nutrition is the production of products that preserve the properties of raw materials in an unchanged form and to the maximum extent that make up nutrient deficiency [1].

The most promising is the application of the principles of food combinatorics $[2,3]$ and the creation of products having a multicomponent composition and containing essential components, mainly in a concentrated form. When constructing diets, modern dietetics faces a dilemma: on the one hand, it is necessary to limit the amount of food consumed in order to achieve a correspondence between the calorie intake and energy consumption of the body, and on the other, to eliminate the existing deficiency of food nutrients. The lack of micronutrients from food is a consequence of a decrease in energy expenditure and a decrease in the total amount of food consumed by modern humans. In Russia, the effect of these factors is exacerbated by a decrease in the consumption of animal products due to a decrease in the income of a significant part of the population, the lack of a national habit of regular consumption of a large number of vegetables, and a number of other skills of good nutrition and a healthy lifestyle. At the same time, in modern conditions, the human need for micronutrients not only does not decrease, but, on the contrary, increases significantly [4]. As a result of a number of objective and subjective reasons, the problem of rationalizing nutrition and, accordingly, improving the population is unsolvable due to only an increase in the consumption of natural products and an extensive increase in the volume of food production. This problem requires qualitatively new approaches and solutions [4]. Currently, snacks are widespread in all countries of the world as products of mass consumption by the population at home and not only, as well as in children's and diet food [5]. Promising types of product are already on the market:

- for therapeutic and preventive nutrition aimed at preventing occupational diseases associated with the specifics of work, living conditions, and to replenish energy costs (miners, oil workers, rescuers, athletes, etc.);

- general purpose with high consumer properties, including quick cooking in disposable packaging;

- new types of products, rations, rations and food systems for crews of orbiting space stations and various contingents of the Armed Forces;

- long-term storage with a given composition of microflora based on milk and fruit and vegetable raw materials, fermented with special strains of lactic bacteria [6].

The aim of this work is to develop stages and apply the principles of food combinatorics in creating optimal compositions of dehydrated products for a healthy diet.

To justify the selected research objects, an analysis of the nutritional potential of plant materials of the Saratov region was previously conducted.

\section{Experimental}

As objects of study, two types of snack products were developed mainly from regional plant raw materials of the Saratov region (3 compositions in each form): 
- the first type of snack consists of the components of the pulp of pumpkin, carrot, blackcurrant and orange with zest;

- the second type of snack consists of components of tomato fruits, onions, parsley greens, dill, basil, coriander.

Research methods: Nutritional value was produced by the calculation method - nutritional value was calculated according to the tables of chemical composition and based on these recommendations on the use of vitamins for men and women ( 2 groups of labor intensity). Water absorption at a hydromodule 1: 10 standard conditions. Vitamins and biologically active substances (flavonoids) were analyzed by reverse phase HPLC on a Dionex Ultimate 3000 chromatograph (Thermo Scientific, USA) using a Luna 5 u C18 (2) 100A column, $5 \mu \mathrm{m} 4.6 \mathrm{~mm} \times$ $150 \mathrm{~mm}$ (Phenomenex), USA), serial number 125617-12. Detection was carried out at the following wavelengths: A, E - $265 \mathrm{~nm}$. Analysis time is 15 minutes (for vitamins) and 25 minutes (for flavonoids). The extracts were chromatographed under isocratic elution conditions (Solvent A - methanol, qualifications (Ultra) gradient HPLC grade (JTBaker, Holland), solvent B - acetonitrile qualifications HPLC grade (Panreac, Spain), in a 80:20 ratio. a flow of $1 \mathrm{ml} / \mathrm{min}$, the volume of the injected sample is $20 \mu \mathrm{l}$, the chromatograph was Controlled and the data was analyzed using the Chromeleon software version 7.1.2.1478 (Thermo Scientific, Dionex, USA).

The calculation method for determining solids or moisture was carried out according to GOST 28561, the analysis of texture indicators was performed on a STZ Texture analyzer (Brookfield, USA). A sample of TA 5 (cylinder D. $12.7 \mathrm{~mm}$ ) was used. The boundary of the relative error of the test method, $\Delta \pm 10 \%$. Organoleptic evaluation was carried out by the method of organoleptic analysis (profile method) GOST R 53104-2008.

\section{Results and discussion}

To determine the nutritional potential of raw materials, developed combined snack products, several benchmark assessment points were identified:

- the quality and quantity of nutrients of raw materials of functional importance (dietary fiber, vitamins and vitamin-like substances, minor components of food with complex effects on the body);

- a combination of organoleptic characteristics, with the possibility of mutual amplification and the formation of a sensory bouquet acceptable to the consumer;

- structural and mechanical properties, allowing the formation of crushed raw materials in the snack before dehydration, and to provide easy chewing after dehydration.

Analysis of the nutritional potential of plant raw materials in the Saratov region showed that the following combinations of raw materials are promising as the basis for snack products:

1 - pulp of pumpkin, carrot, blackcurrant and orange with zest;

2 - tomato fruits, onions, parsley greens, dill, basil, coriander.
Two types of developed snacks were designed (six of the most optimal in composition samples that differ in the proportions of each of the components).

For further research, coded names are assigned to product samples:

- according to the first composition - VFB (vegetables, fruits, berries);

- according to the second composition - VV (vegetables).

In calculating the predicted nutritional value, the mass of the product after dehydration was taken into account. The selection of adaptogenic plant materials, the calculation of acceptable ratios made it possible to predict the replenishment of the body's needs (Tables 1 and 2).

Table 1. The forecasted replenishment of the need for the necessary substances for the composition of VFB.

\begin{tabular}{|c|c|c|c|}
\hline \multirow{2}{*}{ Substances } & \multicolumn{3}{|c|}{ Meeting needs, \% } \\
\cline { 2 - 4 } & $\boldsymbol{V F B 1}$ & $\boldsymbol{V F B 2}$ & $\boldsymbol{V F B 3}$ \\
\hline Alimentary fiber & 37 & 35 & 36 \\
\hline$\beta$-carotene & 79 & 82 & 71 \\
\hline Vitamin A & 14 & 12 & 17 \\
\hline Vitamin E & 16 & 17 & 16 \\
\hline Vitamin PP & 6 & 8 & 7 \\
\hline Vitamin C & 53 & 50 & 54 \\
\hline Flavonoids & 25 & 26 & 23 \\
\hline Phenolic acids & 61 & 99 & 87 \\
\hline
\end{tabular}

Table 2. Predicted replenishment of the need for the necessary substances for composition VV.

\begin{tabular}{|c|c|c|c|}
\hline \multirow{2}{*}{ Substances } & \multicolumn{3}{|c|}{ Meeting needs, \% } \\
\cline { 2 - 4 } & $\boldsymbol{V} \boldsymbol{V} \mathbf{1}$ & $\boldsymbol{V} \boldsymbol{V} \mathbf{2}$ & $\boldsymbol{V} \boldsymbol{V} 3$ \\
\hline Alimentary fiber & 51 & 51 & 50 \\
\hline Vitamin A & 15 & 15 & 15 \\
\hline Vitamin E & 3 & 2,5 & 3 \\
\hline Vitamin K & 110 & 88 & 131 \\
\hline Lycopene & 127 & 126 & 126 \\
\hline Manganese & 3,5 & 3,1 & 4,5 \\
\hline Rubidium & 12 & 16 & 13 \\
\hline
\end{tabular}

The selection of adaptogenic plant raw materials, the calculation of acceptable ratios made it possible to predict the replenishment of the needs of the human body for the following substances: vitamin A from 12 to $15 \%$, while the estimated amount of provitamins $A$, such as $\beta$ carotene and lycopene, compensates for this need significantly from 79 to $127 \%$ of daily allowance, vitamin $\mathrm{K}$ from $88 \%$ to $131 \%$, native dietary fiber from $35 \%$ to $51 \%$ of the recommended daily allowance, depending on the ratio of components in the predicted compositions.

The preservation of essential substances [7-9] will be directly related to the selected method of mechanical processing and thermophysical effects. This conclusion allowed us to propose several methods of mechanical action. The grinding of the components was selected based on the structure of the tissues of each component of the raw material, which would maximize the safety of the cell and avoid the loss of water-soluble essential substances in the grinding process.

To reduce the loss of the most unstable substances under the heat-physical effect during the dehydration 
process, stabilization of the main essential substances is necessary. This is possible due to the diffusion through the permeable cell membranes of antioxidant substances of the feedstock components. In the technology for the production of snacks before dehydration, an exposure of the composite mixture for 10-15 minutes is provided. Since structure-forming substances (sugar, syrups, fats and polysaccharides) are not intended for forming snacks, exposure of the composite mixture with subsequent prepressing will allow for adhesion between the particles of crushed raw materials, continuing the process of interpenetration of active substances through the cell membrane. This process is also intended to preserve the individual organoleptic properties of each ingredient of the composition separately.

A dehydration method was selected that meets the requirements for the preservation of biologically active substances: the rate of dehydration, minimal loss of active components, the immutability of the native properties of raw materials (preservation of the structure of the cell membrane, organoleptic characteristics inherent in raw materials), acceptable to the consumer structural and mechanical characteristics. The use of a step-by-step method of IR dehydrogenation, which provides stepwise removal of moisture with different binding energies, is proposed.

Thanks to the detailed justification of the stages of the technological process for the production of combined snack products using the principles of food combinatorics, it was possible to obtain confirmation of the hypothesis about designing a new type of combined dehydrated products for a healthy diet.

In the study of ready-made dehydrated samples for moisture absorption (Fig. 1), indicating the ability to restore cells, it was noted that under standard conditions, the product swells 5 times in volume.

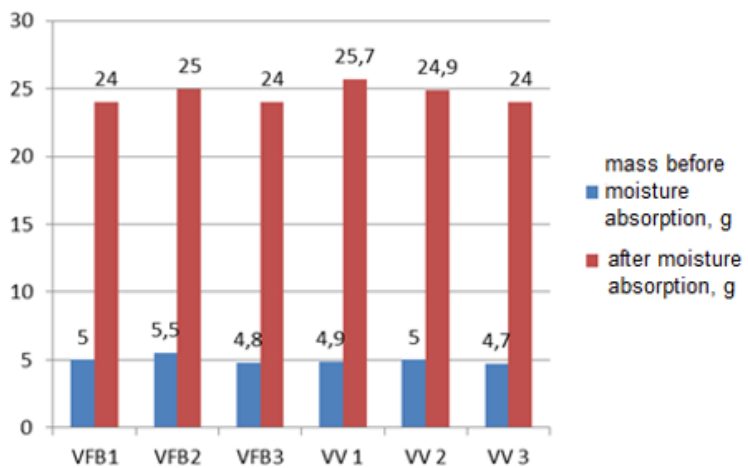

Fig. 1. Change in mass of samples upon absorption of moisture.

During dehydrogenation, flavonoids are stored in sufficient quantities (Fig. 2) and depend on the degree of grinding of the raw material before heat-physical exposure. Oscillations of $6 \%$ in safety between samples are caused by precisely different mechanical methods. The data obtained prove the effectiveness of the application of the food combinatorics principles to create dehydrated food systems designed for healthy nutrition.

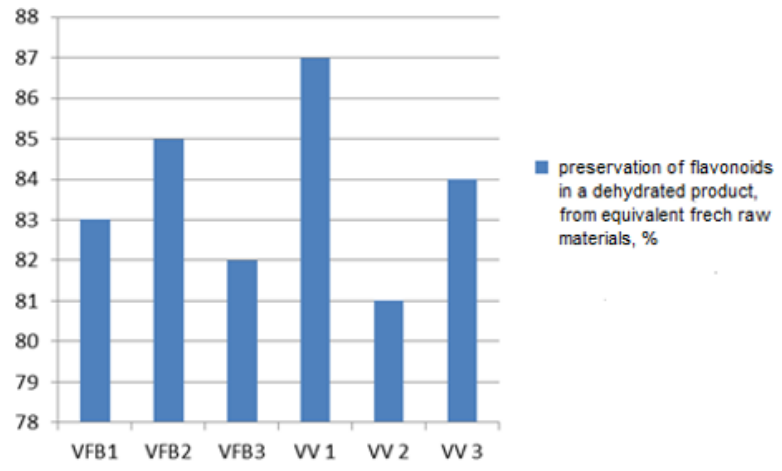

Fig. 2. Preservation of flavonoids after dehydrogenation.

Fig. 3 and 4 show the structural and mechanical characteristics of the samples, which make it possible to judge the force during chewing. It should be noted that VFB samples need more effort to chew, but this is objectively explained by the component composition and assumed. VV samples have a larger number of faults, which indicates a greater fragility of the snack product.

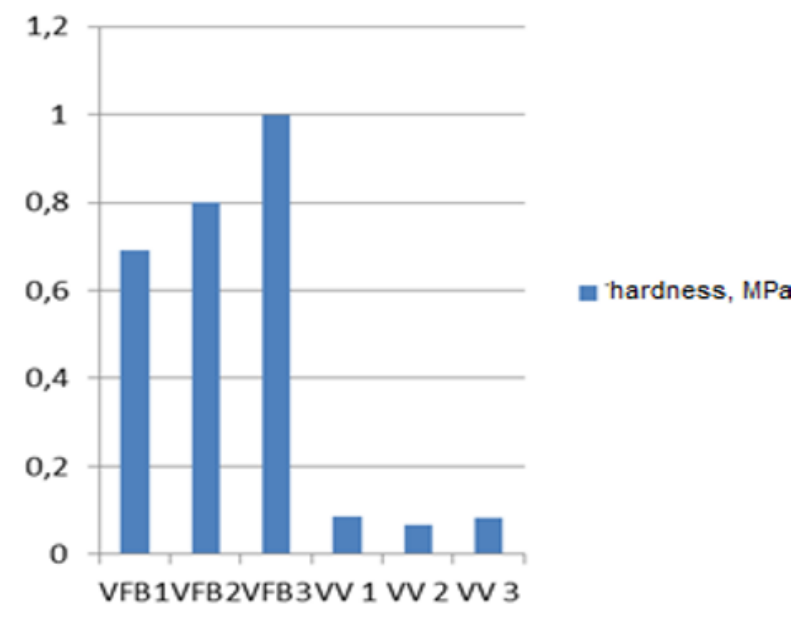

Fig. 3. Hardness of samples.

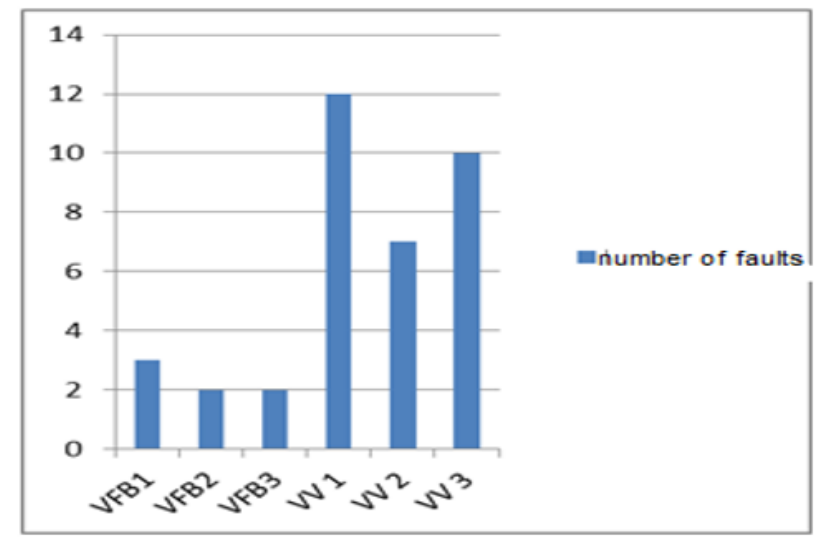

Fig. 4. Number of faults.

All studies conducted, including organoleptic, allow us to conclude that consumer characteristics and developed products are acceptable. And the generalized technological scheme shown in Fig. 5 can be applied to other combinations of raw materials. 


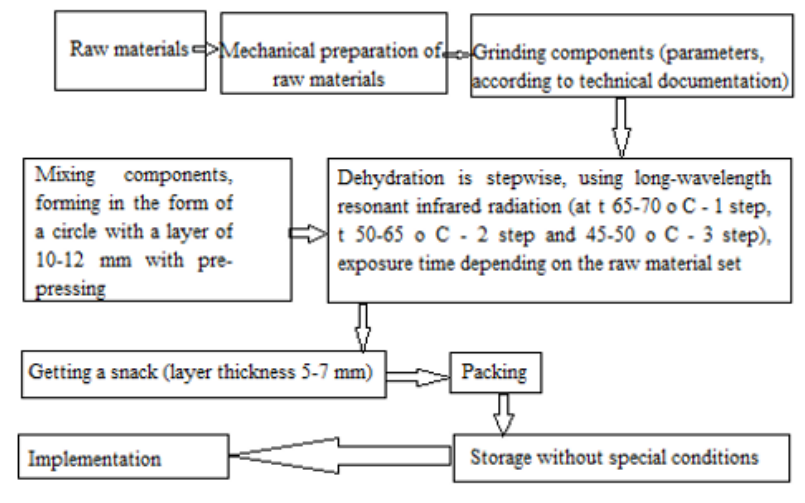

Fig. 5. Technological scheme for the preparation of snacks.

\section{Conclusion}

The application of the principles of food combinatorics in the development of technology for creating optimal compositions of dehydrated products makes it possible to obtain systems of concentrated components of essential substances without significant changes in native properties. And the adopted algorithm can be used to create analog products from other vegetable, fruit and berry raw materials, taking into account the characteristics of the composition and emerging properties. This allows us to consider these technological solutions universal for the development of a wide range of products.

\section{References}

1. Diet, nutrition and the prevention of chronic diseases: report of a Joint WHO / FAO Expert Consultation, WHO Technical Report Series, 916 (World Health Organization, Geneva, 2003)
2. L. Nadtochy, Food combinatorics: Textbook (ITMO University, St. Petersburg, 2016)

3. A. Zaporozhsky, Implementation of the principles of food combinatorics and the substantiation of new biotechnological solutions in the technology of products of herodietic prescription: the dissertation of a doctor of technical sciences (Kuban State Technological University, Krasnodar, 2009)

4. V. Spirichev, L. Shatnyuk, and V. Poznyakovsky, Food fortification with vitamins and minerals. Science and Technology (Sib. Univ. Publishing House, Novosibirsk, 2005)

5. A. Doronin, Functional food products (DeMi Print, Moscow, 2009)

6. On approval of recommendations on rational food consumption standards that meet modern requirements for a healthy diet, Order of the Ministry of Health of Russia dated August 19, 614 (2016)

7. V. Strizhevskaya., I. Simakova., M. Pavlenkova, S. Nemkova, and N. Nosacheva, Development of bioactive combined snack products with innovative dehydration techniques, Science Week SPbPU: Proceedings of the scientific-practical conference with international participation, The Higher School of Biotechnology and Food Technologies,

19-24 November, St. Petersburg, Russia, 184-187 (2018)

8. V. Strizhevskaya, I. Simakova, M. Pavlenkova, S. Nemkova, and N. Nosachyova, E. Wolf, Possibility and Prospects of Preservation of Minor Components in Technology of Fruit Raw Materials Conservation, Biosistems Engineering 2019, 10th International conference, 2082-2088 (2019)

9. V. Strizhevskaya, I. Simakova, and M. Pavlenkova, New Technol., 47, 178-188 (2019) 